\title{
A Dog Tail for Utility Robots: Exploring Affective Properties of Tail Movement
}

\author{
Ashish Singh and James E. Young \\ Department of Computer Science \\ University of Manitoba \\ Winnipeg, MB, Canada R3T 2N2 \\ \{ashish, young acs $_{\text {. umanitoba.ca }}$
}

\begin{abstract}
We present a dog-tail interface for utility robots, as a means of communicating high-level robotic state through affect. This interface leverages people's general knowledge of dogs and their tails (e.g., wagging means happy) to communicate robotic state in an easy to understand way. In this paper, we present the details of our tail construction, and the results of a study which explored a base case of people's reactions to the tail: how various parameters of tail movements and configuration influence perception of the robot's zoomorphized affective state. Our study indicated that people were able to interpret a range of affective states from various tail configurations and gestures, and in summary, we present a set of guidelines for mapping tail parameters to intended perceived affective robotic state.
\end{abstract}

Keywords: human-robot interaction, animal-inspired interfaces, affective computing.

\section{Introduction}

As robots continue to enter people's spaces and environments it will be increasingly important to have effective interfaces for interaction and communication between the people and robots. One such aspect of this communication is people's peripheral awareness of robots' actions and motions, a communication channel which has proven important for human-human interaction in work roles similar to utility robots; for example, as office assistants, robots should be as non-intrusive as possible yet still provide ambient awareness of their tasks $[10,32]$. In this paper we introduce the use of a dog tail for robots as a peripheral awareness communication mechanism (Fig. 1).

Dogs are one of the most preferred pets in many countries around the world, and as such we posit that even non-dog owners have a passing knowledge of dogs from the general "social stock of knowledge" [4, 14]. Thus we believe that dogs' tail gestures and vocabulary are generally well understood, at least on a basic level, and that utility robots can leverage this to unobtrusively broadcast various aspects of their state to people while they work. For example, a cleaning robot could keep its tail high on a full battery and slowly lower it to indicate less energy, it could lower its tail (between its wheels) to indicate a stuck wheel, or a delivery robot could wag its tail quickly to 


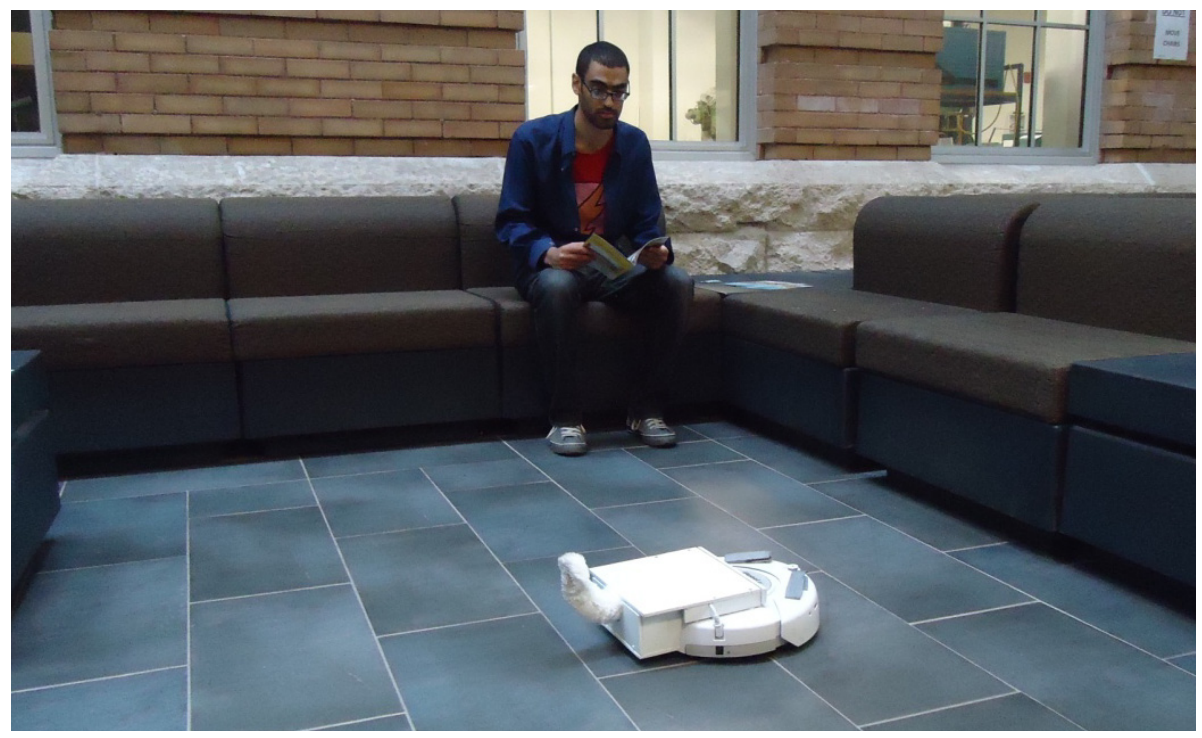

Fig. 1. A person notices the ambient tail state of a passing robot

indicate it found the person it was looking for. This tail interaction would further leverage people's existing tendencies to anthropomorphize and zoomorphize utility robots, for example, people give them names and buy them clothes [7, 30].

Affect is easily recognized by people (at least at the abstract level) and utility robots that find themselves collocated with people can use this communication channel. While a tail would enable a robot to broadcast zoomorphic affective information, it is not clear how this information will be interpreted by people in relation to its state; for one, a real dog uses its tail in concert with the rest of its body language, while our utility robot uses the tail in isolation. In addition to building a utility-robot dog-tail prototype, we conducted an experiment that explored how people respond to the tail in general, and how various tail parameters (e.g., wagging speed or height) impact the robot's perceived affective state. From this we summarize our findings into a toolkit set of guidelines for developing dog-tail interfaces to broadcast desired state information. The contributions of this paper are: a) a novel utility-robot dog-tail interface design and implementation, and b) a set of design guidelines, grounded in a study, for developing dog-tail behaviors for specific affective response.

\section{Related Work}

Part of the affective computing tradition in human-computer interaction is to incorporate human or animal-like affect and emotion directly into interfaces [20, 21], for example, a picture frame which uses an ambient color display to communicate emotion between people when they are apart [5]. There is a well-established 
application of ideas from affective computing to human-robot interaction, where impressions of robotic affect can be used to help users gain high-level state information without requiring them to read complex sensory information $[6,11,12$, 31]. Some have suggested the use of facial expressions and embodied gestures, where examples include mechanized faces with eyebrows, mouths, etc. [1-3, 34], animated faces on screens $[16,18]$, using mixed reality to superimpose graphics faces on robots [31], human-like whole gestures with arms, etc. [2], or even using gaze [29]. More abstract methods such as colors and sounds have similarly been used for communicating emotion to represent state. For example, both the Breakbot and AIBO robots use color in this way, and the Sony AIBO further uses puppy-like sounds [8, 23]. Our work follows this overall approach by using affect and emotion, via a robotic tail, to encourage attribution of affect for the purpose of conveying high-level robot state.

Animals have commonly been used for robotic interface inspiration. Leonardo, for example, was designed as a fantastical mammalian creature that people can relate to and communicate with [2], the Sony AIBO was designed explicitly to act as a puppy [8], and one robot can follow its owner on a leash similar to a dog [33]. Several robots have also used tails in concert with other features to help communicate with people, to convey emotions as part of their animal persona or design $[8,26]$. In our work, we investigate how a dog-inspired tail interface can be applied to utility robots, and how people perceive the affective impact of its various motion possibilities.

Zoological research tells us that dogs can convey a broad range of states through their tails, for example, suggesting a happy state by wagging, high arousal or selfconfidence by raising their tails, or fear by lowering their tail $[1,5]$. Robotic pets such as the AIBO only use simple tail wagging [8], and it is still unclear how a wide range of behaviors can scale to robots. One study discovered that a tail can invoke memories and that interpretations vary with wag speed in one dimension [27], although this work did not aim to formally parameterize the tail motions and measure affect as we do, and also did not find consistent results across people. Further, robotic tails could produce motions not found with real dogs, such as moving in a full circular motion. In our work, we extend tail movement capabilities and formally investigate how people perceive a full range of motions in terms of affect.

\section{Implementation}

We based our dog-tail interface implementation (Fig. 2) on a technique used in hobbyist animatronics [28, 35] where the tail was constructed from a modified common construction toy kit (Klixx): the interlocking pieces were sanded to achieve smooth movement and to increase range of motion, and paperclips were inserted through drilled holes to strengthen the joints (Fig. 3). Tail deformation was achieved using a two-dimensional cable and heavy-duty servo pulley mechanism attached to a wooden board. The cables were attached to the tail by being threaded through the paper clips. 


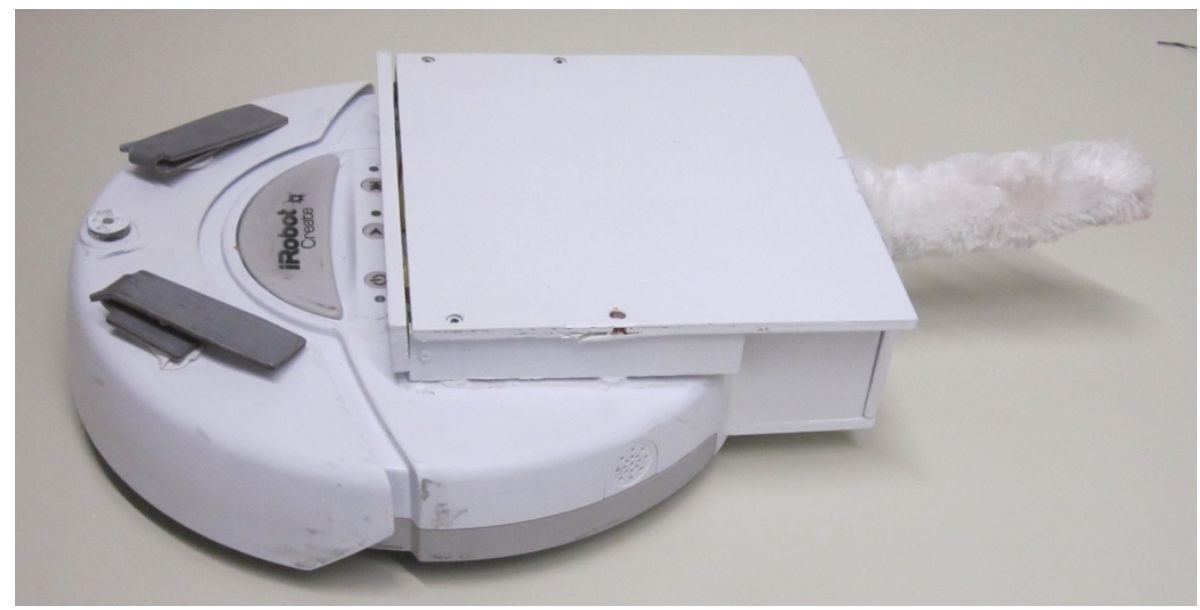

Fig. 2. An iRobot Create with our dog-tail attached
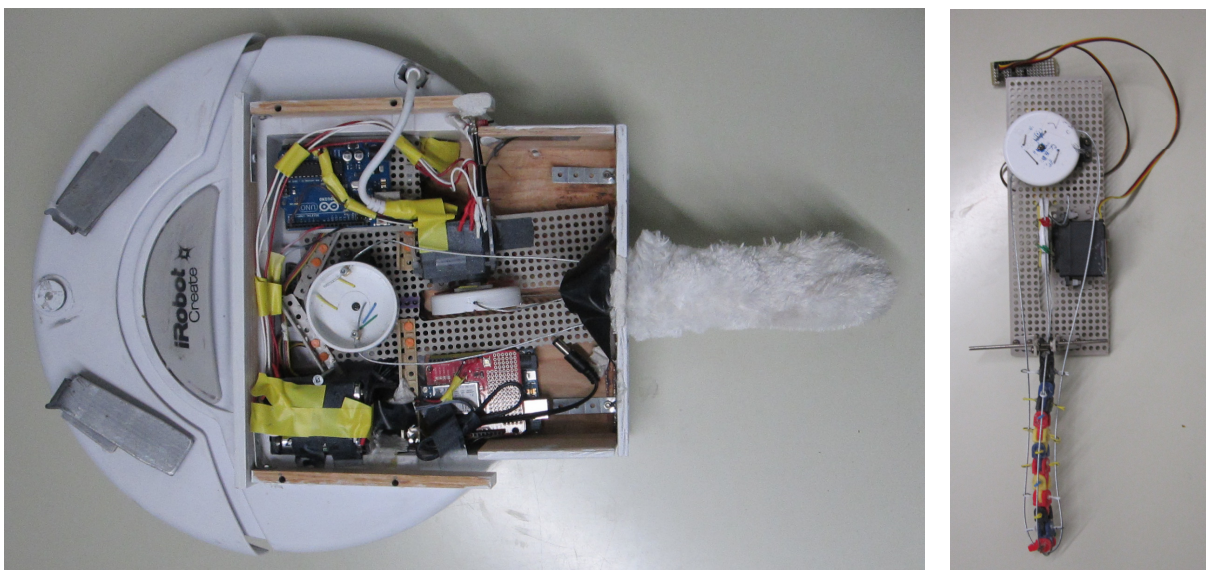

Fig. 3. Top-view of the tail assembly and before putting it inside the wooden box (right)

A key point with this mechanism is that we had to ensure that the two mounted servos were directly in line with the central tail axis, both in terms of height above the platform and lateral offset; slight alignment deviations added a tilt to the tail movements, making direct left-right or up-down movements difficult, and larger deviations resulted in the tail twisting (crumpling).

The entire tail mechanism was mounted on an iRobot Create disc-shaped robot, covered with a wooden box to hide the internal workings, and the tail itself was covered with white furry fleece (taken from a stuffed animal) to improve the appearance of being a dog tail.

The electronic implementation was achieved by using two Arduino Uno prototyping platforms, one to control low-level tail behavior and one to drive the robot around a space, and a WiFly wireless internet module for remote control. 
The basic tail design was determined through informal pilot studies (5 participants). We experimented with tail coverings: no covering was seen as too mechanical or unpleasant, a white spandex (hose) cover was seen as somewhat reptilian and left a negative impression, and our fur covering was seen as cute and fun. Despite worries that fur would be out of place with a plastic robot, this was not a strong concern in the pilot. We tested tail length in terms of now natural it appears on our robot; we built short $(10 \mathrm{~cm})$, medium $(15 \mathrm{~cm})$, and long $(32 \mathrm{~cm})$ versions. The medium length was favored strongly in the pilot and used in our final version.

\section{$4 \quad$ Exploratory Methodology}

Zoological research tells us much about how dogs use their tail to communicate [9, 13], and we believe that people have some basic understanding of dog-tail communication from the general social stock of knowledge [10], for example, that wagging is a positive or playful state. However, we did not yet know if this knowledge transfers to interacting with a robot via a dog-tail. We also did not know to what extent people understand what a tail may be trying to communicate, or how different kinds of tail motions will be interpreted by people. As an initial step, through pilots we observed that people do not (at all) understand intricate dog-language specifics, e.g., different meanings when wagging on the left vs. the right. However, in the pilot people understood well that dog tails are used for communication, and could understand basic language such as wagging and raising or lowering the tail. Our exploratory goal is to leverage this general understanding (i.e., we do not want to require people to have training) and to systematically explore how people interpret a full range of tail motion possibilities: we implemented a broad range of tail motions and configurations, at different speeds, wag-sizes, and so forth, and conducted a study to evaluate how people perceive the resulting robotic affective states.

\subsection{Measuring Perceived Affect}

To classify people's perceptions of the robot we applied Russell's Circumplex Model of Affect, a standard psychological model of affective and emotional states [17, 24]. This model breaks affect into two scales or dimensions: valence (pleasure), from displeasure to pleasure, and arousal, from low to high energy.

To measure people's perceptions of the robot's affect we employed the SelfAssessment Manikin (SAM) [19]. SAM is a standard psychological instrument for rating affective states on the above affect model, where valence and arousal are represented by a series of easy to understand comic-like pictorial representations: from a very unhappy to a very happy character on the valence dimension, and from a sleepy low-energy to a high-energy awake character on the arousal dimension. People can rate an affective state simply by selecting the most appropriate pictorial on each dimension; in our case we used seven-point scales. Although generally used for a person to rate their own feelings, this method can also be used to rate the perceived state of others [22]. 


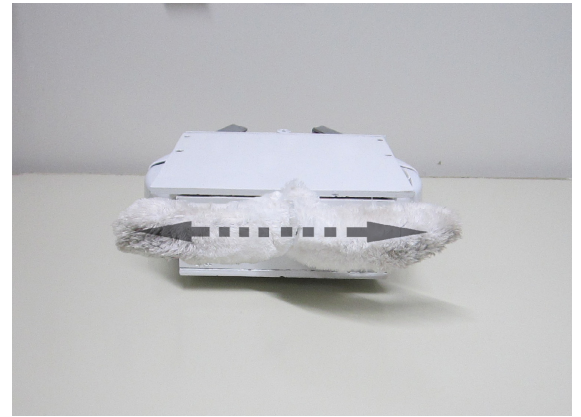

a) Horizontal wagging

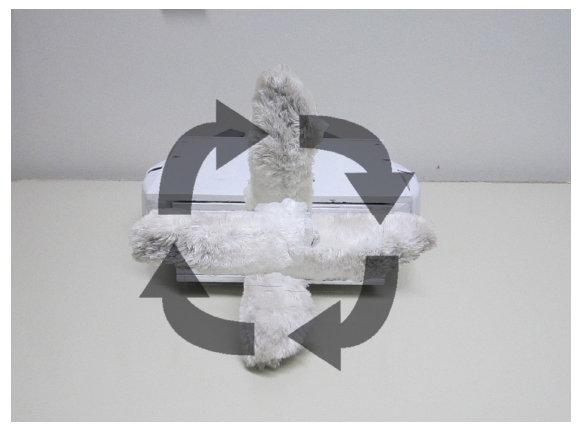

c) Circular wagging

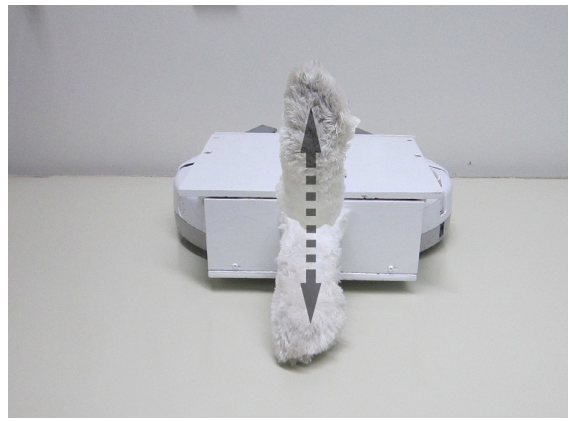

b) Vertical wagging

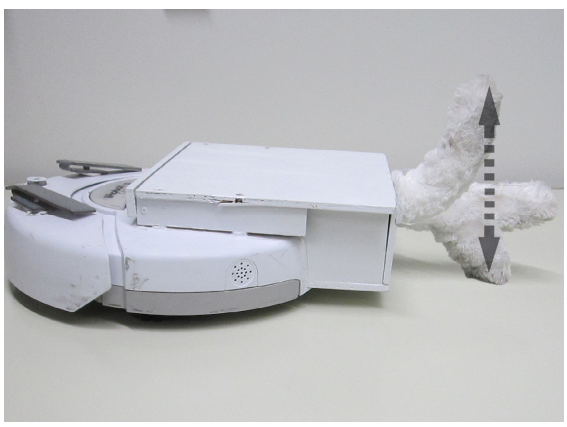

d) Side-view of robot, in this case vertical wagging

Fig. 4. The three continuous motion patterns used in our study

As part of our analysis, we correlate our observed valence and arousal with previous work on classifying emotions on these scales [19]. This provides a means to generate descriptive keywords that are capable of explaining how people may interpret the particular motion.

\subsection{Exploring Tail Vocabulary}

In developing a broad range of tail motions and states, we used nature-inspired possibilities (how real dogs act) as well as additional possibilities enabled by our tail configuration but not used in nature. We categorize our selected tail settings into: continuous wagging, the tail is always moving, gestures, the tail does an action at a certain time, and postures, the tail maintains a static state.

We developed three forms of wagging: horizontal, where the tail moves left and right on a plane roughly parallel to the floor similar to as in nature [9, 13] (Fig. 4a), vertical, where the tail moves up and down perpendicular to the floor (not found in nature, Fig. 4b), and circular, where the tail moves in a complete circle (not found in 
Table 1. The full range of tail configurations we developed and tested

\begin{tabular}{cll}
\multicolumn{1}{c}{ category } & sub-type & \multicolumn{1}{c}{ attributes } \\
\hline \multirow{2}{*}{ continuous wagging } & horizontal & $\begin{array}{l}\text { speed: low, medium, high } \\
\text { wag-size: small, medium, large } \\
\text { height: low, parallel to floor, high }\end{array}$ \\
\cline { 2 - 3 } & vertical & $\begin{array}{l}\text { speed: low, medium, high } \\
\text { wag-size: small, medium, large }\end{array}$ \\
\cline { 2 - 3 } & circular & speed: low, medium, high \\
\hline \multirow{2}{*}{ action gestures } & raising & $\begin{array}{l}\text { speed: low, medium, high } \\
\text { height: low, high }\end{array}$ \\
\cline { 2 - 3 } & lowering & $\begin{array}{l}\text { speed: low, medium, high } \\
\text { height: low, high }\end{array}$ \\
\hline static postures & & height: low, medium, high \\
\hline
\end{tabular}

nature, Fig. 4c). All three types were created at low, medium, and high speeds. We varied horizontal and vertical to have either a small, medium, or large wag-size (how tight or wide the wag was, no change in speed). Finally, we varied horizontal wagging to have a low, parallel, or high offset in relation to ground.

We developed two tail gestures, a raising and a lowering action, to mimic how dogs act in nature: the tail was kept at a non-moving neutral state slightly below center (as with a real dog) except when it moved to complete a gesture. The tail would hold the gesture for 0.5 seconds before returning to the neutral state. We created low, medium, and high speed versions of the gestures, referring to the time taken to change from neutral to target state (raised or lowered), and a low and high offset version of each, representing how far the gesture moved from neutral. Finally, we had three static postures: a low tail, a straight tail parallel to the floor, and a high tail.

Table 1 presents an overview of our 31 motions deriving from the above configurations. Note that attributes are manipulated independently of others and thus some entries of the table are identical. For example, for the three wag sizes of horizontal wag, the other two attributes (speed, height) were kept fixed; for horizontal wag, the medium speed and the medium height settings were effectively identical. This reduction yielded 26 unique behaviors that were shown to participants.

\subsection{Anticipated Interpretations}

In general, we assume that high tail height will have high valence, and that valence values will decrease when the height is decreased, as this is naturally how dogs communicate with their tails $[9,13]$. Additionally, based on previous motion and emotion work we expect that, in general, higher speeds will have higher arousal [25]. We expect this to happen for wagging, gestures, and postures. 


\section{Study}

We recruited 20 participants from our local university population to participate in our study: 12 males / 8 females, aged 18-47 (M=24.25, $\mathrm{SD}=6.79)$. Our study was reviewed and approved by our university research ethics board, and all participants received $\$ 10$ for their participation in the 60 minute study.
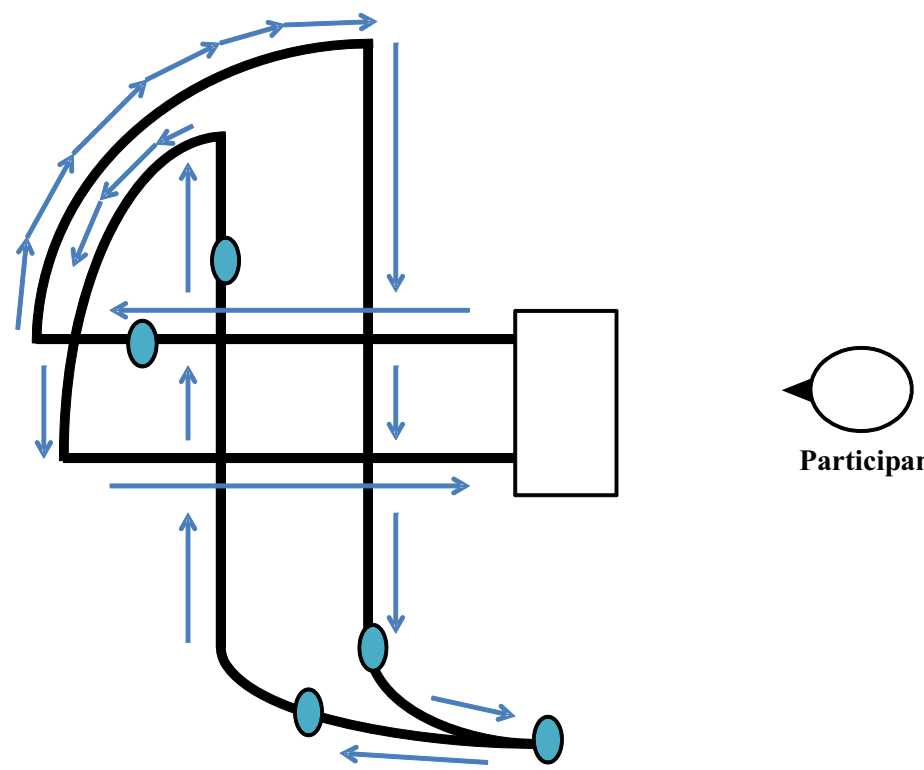

Participant
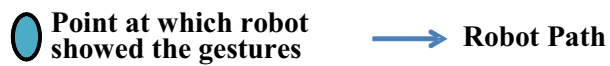

Robot base (start/end point for each motion)

Fig. 5. Robot motion plan, blue ellipses define the points where robot showed action gestures

Participants were brought to our lab environment, and after a brief introduction, signed an informed consent form and received their compensation. We introduced the robot and the tail, the concept of the robot using the tail to communicate mood, and introduced the SAM scales based on its recommended text [15]. Participants proceeded to view the tail behaviors, order of appearance counterbalanced across participants. Participants were given 15 seconds post-demonstration of each tail behavior to rate the configuration on the SAM scales. Finally, we conducted a semistructured interview, to investigate general views on the tail interaction, and debriefed the participants before ending the study. All studies and interviews were videotaped.

The layout of the study environment is shown in Fig. 5, where the participant was seated at a desk positioned to easily view the robot's motion, as it followed the path indicated. We designed this path to provide views of the tail from the front, sides and behind. The robot used the same path for all tail configurations, where the tail action 
was the only thing that changed. Blue ellipses on the robot path represent the spots where the robot showed the action gestures such as "raising the tail" which can only happen at certain points. We used side and back views of the robot for showing action gestures so as to provide a clear view of the tail to the participants; we did not have a view from the robot's front as the robot might have occluded the tail. Other than action gestures, all tail motions were programmed to initiate when the robot started to move and were stopped when robot came to a halt. The path took 35 seconds to complete, after which the experimenter returned the robot to its original position to minimize drift over the cases.

\subsection{Results}

We performed six primary analyses based on our configurations highlighted in Table 1 and our anticipated interpretations; our dependent variables were the participant ratings of affect on the valence and arousal dimensions.

Speed vs. Wag Type. We conducted a 2 way ANOVA on wag type (horizontal, vertical, circular) versus speed (low, medium, high). As the assumption of sphericity was violated for the main effect of speed on valence (Mauchly's test, $\mathrm{X}_{2}{ }_{2}=14.93$, $\mathrm{p}<.05)$, degrees of freedom were corrected using Greenhouse-Geisser estimates of sphericity (e=.631).

All effects are reported as significant at $\mathrm{p}<.05$. There was a significant main effect of the wag speed on both valence $F_{1.26,24.30}=9.79$ and arousal $F_{2,36}=71.38$. Planned contrasts (we predicted that more speed would express more energy and more positive valence) revealed that, on both the valence and arousal dimensions, high speeds were rated significantly higher than medium speeds $F_{1,18}=18.53$ valence, $F_{1,18}=42.92$ arousal, and low speeds $\mathrm{F}_{1,18}=11.79$, valence, $\mathrm{F}_{1,18}=99.42$, arousal.

There was also a significant main effect of wag type on both valence $F_{2,36}=15.52$ and arousal $\mathrm{F}_{2,36}=39.63$. Post-hoc tests (with Bonferroni correction) reveal that vertical wagging $(\mathrm{M}=-0.56, \mathrm{SD}=1.49)$ was rated as lower valence than both horizontal $(\mathrm{M}=1.44, \mathrm{SD}=0.98)$ and circular $(\mathrm{M}=0.98, \mathrm{SD}=1.83)$, although there was no difference found between horizontal and circular. For arousal, all differences were significant: horizontal wagging $(\mathrm{M}=0.63, \mathrm{SD}=1.3)$, vertical $(\mathrm{M}=-0.56, \mathrm{SD}=1.45)$, and circular $(\mathrm{M}=1.61, \mathrm{SD}=0.97)$. These relationships are shown in Fig. 6.

There was a significant interaction effect between the wag type and speed on valence $\mathrm{F}_{4,72}=3.74$ and arousal $\mathrm{F}_{4,72}=3.02$, indicating that speed's effects on perceptions of valence and arousal depends on the wag type. For valence, post-hoc tests (with Bonferroni correction) revealed that all three speeds yielded different results for horizontal wag, but no significant effects were found for vertical or circular wags, as suggested by Fig. 6. For arousal, post-hoc tests (with Bonferroni correction) reveal that speed is a significant predictor of measured arousal for horizontal and vertical wagging, but for circular wagging, low speed is significantly different from medium and high, which themselves are not different. 


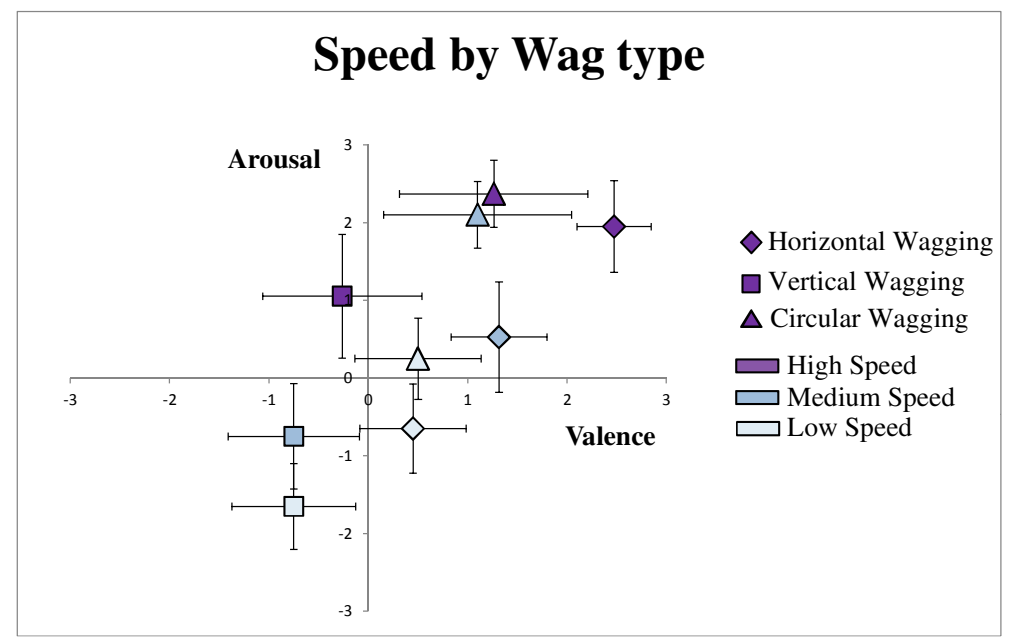

Fig. 6. Speed by Wag Type, error bars show $95 \%$ confidence interval

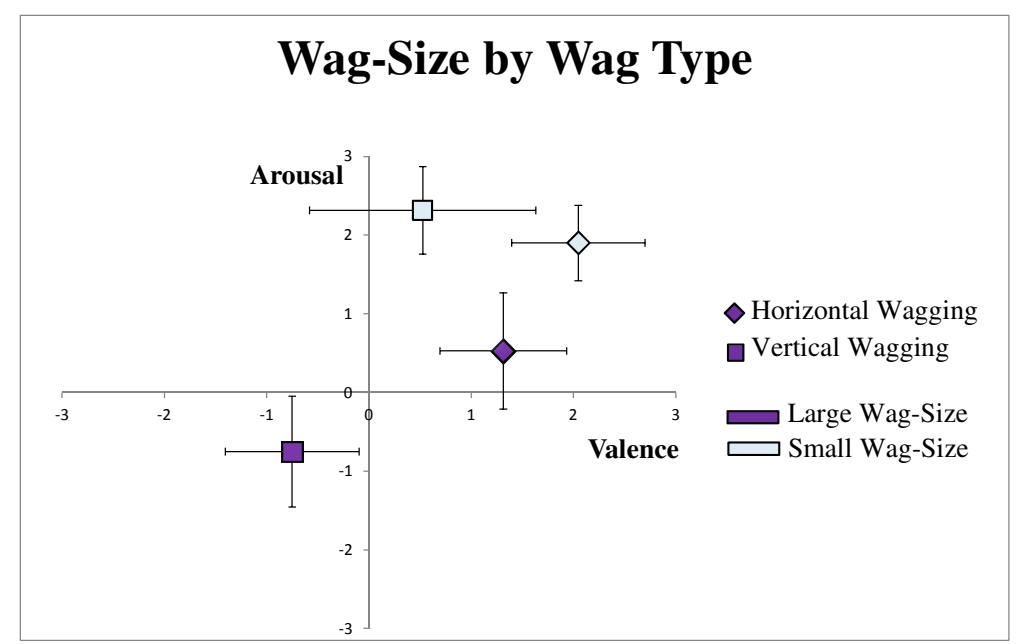

Fig. 7. Wag-Size by Wag Type, error bars show 95\% confidence interval

Wag-Size vs. Wag Type. We conducted a 2-way ANOVA on wag-size (small and large) versus wag type (horizontal, vertical); all effects reported significant at $\mathrm{p}<.05$. There was a significant main effect of wag-size on both valence $F_{1,17}=7.77$ and arousal $\mathrm{F}_{1,17}=48.39$, showing that smaller wag-size increases perception of both valence and arousal (Fig. 7). A significant interaction effect was found between the wag type and how different wag-size were perceived for arousal $F_{1,17}=12.19$ indicating that small wag-size is significantly different from large wag-size in the arousal dimension. 


\section{Height of Horizontal Wagging}

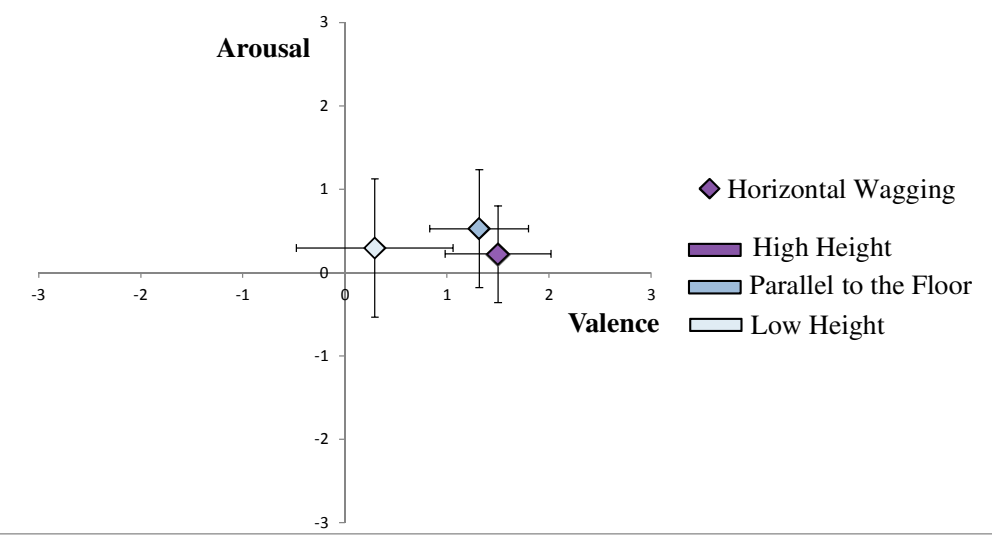

Fig. 8. Height of Horizontal Wagging, errors bars show 95\% confidence interval

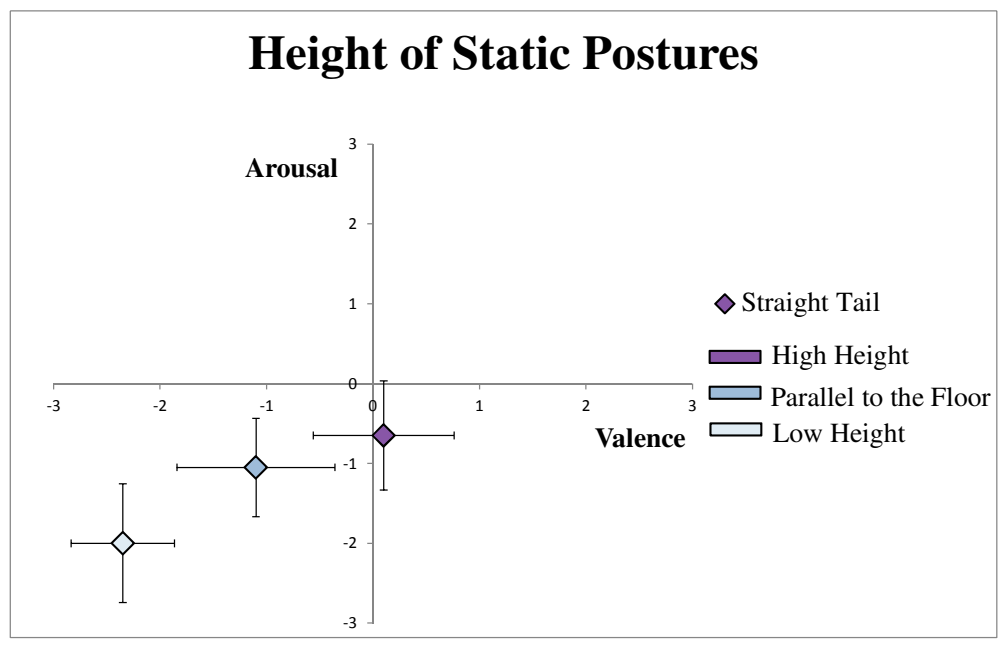

Fig. 9. Height of Static Postures, error bars show 95\% confidence interval

Height of Horizontal Wagging. We conducted a 1-way ANOVA on height (low, parallel to floor, high) with horizontal wag type (3 levels of wagging); all effects reported significant at $\mathrm{p}<.05$. There was a significant main effect of height on valence $\mathrm{F}_{2,32}=6.601$, with planned contrasts highlighting that both medium $\mathrm{F}_{1,16}=4.69$ and high height $\mathrm{F}_{1,16}=12.48$ were higher valence than low height. This indicates that high height had more valence than low height (Fig. 8). There was no effect on arousal. 
Height of Static Postures. We conducted a 1-way ANOVA on height (low, parallel to floor, high) with static postures (3 levels of straight tail); all effects are reported significant at $\mathrm{p}<.05$. There was a significant main effect of the height on perceived valence $F_{2,38}=21.4$ and arousal $F_{2,38}=6.36$. Planned contrasts for valence showed that low height $(\mathrm{M}=-2.35, \mathrm{SD}=1.04)$ was lower rated than both medium $(\mathrm{M}=-1.1$, $\mathrm{SD}=1.59)$ and high $(\mathrm{M}=-0.1, \mathrm{SD}=1.41)$, and for arousal low height $(\mathrm{M}=-2.00$, $\mathrm{SD}=1.59)$ was lower rated than high $(\mathrm{M}=-0.65, \mathrm{SD}=1.46)$ (other contrasts nonsignificant). This explains that high height had more arousal and more valence as compared to low height (Fig. 9).

Non-significant Tests. No significant effects were found using ANOVAs on speed (low, medium, high) by action gestures, or height (low, parallel to the floor, high) by action gestures.

Semi-structured Interview. From the post-study semi-structured interview, we found that: 17 participants (85\%) zoomorphized the robot, for example, saying "it looks like an animal," "it felt like a dog." Additionally 2 female participants (25\% of females) asked the name and gender of the robot and 6 of them (75\% of females) discussed its "cuteness."

19 participants $(95 \%)$ responded positively when asked if they found the dog-tail interface easy to understand and read, saying such things as "I am able to perceive its feelings," "it was easy to understand feelings of the robot," (we note that participants were introduced to the gestures as "feelings" through the explanation of the SAM affective measurement instrument). Some, however, suggested that we add other dog elements, such as puppy sounds to improve the communication clarity. Many mentioned that they were also interested in seeing the dog-tail interface on other utility robots, and some ( 2 participants) were interested in seeing a cat-tail version.

\subsection{Discussion}

We observed that people readily accepted a dog-tail interface on a utility robot, easily understood the concept of the robot communicating through the tail, and that the tail interface has a broad and detailed communication vocabulary that people can consistently understand. We observed that basic dog-tail language such as higher tail height is understood, and also that higher tail wagging speeds result in perceptions of higher arousal, and in general, also result in more positive perceived valence. This was also echoed in the static tail postures, although with no movement, results were all generally less aroused and less positive than their moving counterparts.

We further found consistent differences in wagging types. While horizontal wagging was generally perceived as positive valence, vertical wagging was seen as being more negative - even with faster wags - and circular was somewhere in between with less clear results on valence. Thus, different wag types can be used depending on what a robot is trying to communicate. We feel that this inclusion of non-natural motions did not hinder our results as designers are free to stick to natural ones, and our statisticallysignificant results (including non-natural motions) indicate that there is a base-line common interpretation between people that can be used in design. 
Upon consideration of our lack of consistent findings for our action gestures, we realized that the robotic motion itself and the neutral tail state held when a gesture was not being performed was a likely confound, where people perhaps rated those constant elements instead of the periodic gesture. This is supported by the fact that the perceived valence and arousal of all action gesture movements were tightly grouped.

One perhaps unexpected result was that smaller wag-sizes result in perceptions of higher valence and arousal; we expected these motions to have lower results given their lower movement profiles. Upon consideration, however, we realized that smaller wag-sizes at the same tail speed will result in more wags per second, perhaps increasing the perception of speed.

Overall, our results show that people were able to understand affective robotic states as conveyed using a tail, and as such this technique could be used as a peripheral-awareness channel for conveying high-level robotic state to people. For example, energetic vs. fatigued tail motions could be used to show battery level (e.g., fast/slow tail wag), or a robot could appear depressed (low-arousal/valence, e.g., slow-moving low tail posture) to show navigational confusion such as being lost. By communicating these abstract states, a utility robot can indicate its present state using people's existing knowledge of dog-tail movement and help them understand when and how they should interact with the robot.

\section{Design Guidelines}

From our results we formed the following tail guidelines to help designers in creating their own dog-tail interfaces that convey affect. We present our guidelines from three perspectives: motion parameters (speed, wag-size and height), wag-type (horizontal, vertical and circular wagging) and postures (straight tail-high and straight tail-low). In addition, we present the results from an exercise where we correlated our average arousal and valence ratings to existing knowledge of affective states on these continuums. We envision that this section will be useful to those creating dog-tail interfaces, aiding them in selecting tail movements to represent a desired affective state that can be understood by people without having to undergo training.

\subsection{Dog-Tail Motion Parameters}

Speed. A higher speed projects a higher valence and arousal (e.g., elated) and a lower speed projects a lower valence and a lower arousal (e.g., uninterested).

Wag-Size. A smaller wag-size projects a more positive arousal (e.g., energetic) and a larger wag-size projects a less arousal (e.g., lazier).

Height. A higher tail projects a more positive valence (e.g., happier), and lower tail a more negative valence (e.g., sadder). 


\subsection{Dog-Tail Wag-Types}

Horizontal Wagging. This is the natural form of wagging, as found in dogs. This type of wagging can convey a range of valence and arousal values, starting from medium to high.

Circular Wagging. A tail wagging in circular motion may be able to project a more positive arousal as compared to horizontal and vertical wagging at the same speeds.

Vertical Wagging. A tail wagging in vertical motion generally projects a more negative valence and a slightly more negative arousal as compared to horizontal and circular wagging, although medium high arousal states can be achieved with high speeds or small wag sizes.

Table 2. Adjectives matching participant ratings of tail motion

\begin{tabular}{|c|c|c|c|}
\hline category & sub-type & parameter & attributes and descriptive keywords \\
\hline \multirow{6}{*}{ continuous wagging } & \multirow{3}{*}{ horizontal } & speed & $\begin{array}{l}\text { low - modest } \\
\text { medium - wondering } \\
\text { high - joyful and elated }\end{array}$ \\
\hline & & wag-size & $\begin{array}{l}\text { small - strong, mighty and powerful } \\
\text { large - interested }\end{array}$ \\
\hline & & height & $\begin{array}{l}\text { low - contempt } \\
\text { parallel to floor - awed } \\
\text { high - wonder }\end{array}$ \\
\hline & \multirow[t]{2}{*}{ vertical } & speed & $\begin{array}{l}\text { low - solemn } \\
\text { medium - shy and disdainful } \\
\text { high - aggressive }\end{array}$ \\
\hline & & wag-size & $\begin{array}{l}\text { small - aggressive } \\
\text { large - selfish and quietly indignant }\end{array}$ \\
\hline & circular & speed & $\begin{array}{l}\text { low - reverent } \\
\text { medium - aggressive and astonished } \\
\text { high - overwhelmed }\end{array}$ \\
\hline \multirow{4}{*}{ action gestures } & \multirow{2}{*}{ raising } & speed & $\begin{array}{l}\text { low, medium and high - shy, selfish, } \\
\text { disdainful or weary }\end{array}$ \\
\hline & & height & $\begin{array}{l}\text { low and high - shy, selfish, disdainful, } \\
\text { weary timid and fatigued }\end{array}$ \\
\hline & \multirow{2}{*}{ lowering } & speed & $\begin{array}{l}\text { low, medium and high - shy, selfish, } \\
\text { disdainful or weary }\end{array}$ \\
\hline & & height & $\begin{array}{l}\text { low and high - shy, selfish, disdainful, } \\
\text { weary timid and fatigued }\end{array}$ \\
\hline static postures & & height & $\begin{array}{l}\text { height: low - lonely } \\
\text { parallel to floor - fatigued } \\
\text { high - concentrating }\end{array}$ \\
\hline
\end{tabular}




\subsection{Static Dog-Tail Postures}

Static dog-tail postures provide more subdued impressions of affect and valence than the moving counterparts. A low, static tail projects a very low valence and arousal, while a higher tail makes this impression more moderate.

\subsection{Correlating Tail Motions to Affective Adjectives}

We correlated our results to existing work that maps data points on the arousalvalence space to affective adjectives [18], as a means of generating loose-yetinformative keywords to roughly describe how various tail configurations may be perceived. We took the average rating for each motion and correlated it with the closest point on the previous work. A summary is given in Table 2.

\section{$7 \quad$ Future Work}

Our work is limited to giving a robot a dog-tail only for communicating different affective states. In contrast, a real dog uses its face, eyes, voice body language, etc., to accompany its tail motions to create more complex expressions for deeper communication. We hope to continue our line of work to investigate what other aspects of dog communication can be used by robots in similar ways, or even which other animals can serve as inspiration for developing this type of interface.

While the aim of this study was to develop an understanding of how a robot may communicate using a dog tail, and how people may perceive the communication, moving forward it will be important to further develop our guidelines to provide researchers with more concrete tools for tail-interface design. For example, although our results and guidelines help designers decide how to communicate a desired affective state, we do not yet address how to move from low-level robotic state (e.g., battery level, malfunction, etc.) to affective ones. While this is a broad question for HRI in general, we believe that we can follow the dog metaphor as one promising direction for developing this kind of mapping.

Currently, we have only placed our dog tail on a small robot that sits close to the ground (similar to a small dog). We will explore how our tail will translate to other morphologies such as a humanoid robot or flying robot, and other domains such as toy robotics. Part of this question will be to explore the limits of use. For example, while we focused currently on utility robots, we will explore other less obvious applications such as inanimate objects (e.g., a printer) to help convey the devices' state, and will consider where the tail interface may not be applicable, for example, for remote control robotics or industrial machines where the tail may not be monitored.

\section{Conclusion}

In this paper we presented an original dog-tail interface and conducted a formal evaluation to investigate how people perceived the affective states of a robot equipped with a dog tail, across a full range of tail behaviors. We found that the tail was able to convey a broad range of affective states and that people reliably interpreted the tail 
motions in a consistent fashion. From this, we summarized our results into design guidelines for creating dog-tail interfaces.

Overall, we anticipate that our contribution of exploring and mapping how robots can use dog tails to communicate affect will be of use to HRI designers, providing them with a new paradigm for robotic communication. Further, we hope that robots using this kind of periphery communication will help people in understanding their state and help in deciding when and how they should interact with the robot.

Acknowledgement. We are thankful to Dr. Jacky Baltes and Andrew Winton for their valuable time and input. We would also like to thank $\mathrm{B}$ and $\mathrm{K}$ Winton Industry for lending their tools to design our interface. Finally, we would like to express our gratitude to Derek Cormier, Masayuki Nakane and Ibrahim Shahin for their support in the study.

\section{References}

1. Breazeal, C.: Emotion and Sociable Humanoid Robots. International Journal of HumanComputer Studies 59(1-2), 119-155 (2003)

2. Breazeal, C., Hoffman, G., Lockerd, A.: Teaching and Working with Robots as a Collaboration. In: Proceedings of the International Joint Conference on Autonomous Agents and Multiagent Systems - AAMAS 2004, pp. 1030-1037 (2004)

3. Van Breemen, A., Yan, X., Meerbeek, B.: iCat. In: Proceedings of the International Joint Conference on Autonomous Agents and Multiagent Systems - AAMAS 2005, p. 143 (2005)

4. Brown, S.E.: Self Psychology and the Human-Animal Bond: An Overview. The HumanAnimal Bond and Self Psychology: Toward a New Understanding 12(1), 67-86 (2004)

5. Chang, A., Resner, B., Koerner, B., Wang, X.: Ishii. H.: LumiTouch. In: Proceedings of the International Conference Extended Abstracts on Human Factors in Computing Systems - CHI 2001, p. 313 (2001)

6. Duffy, B.R.: Anthropomorphism and the Social Robot. Robotics and Autonomous Systems 42(3-4), 177-190 (2003)

7. Forlizzi, J., DiSalvo, C.: Service Robots in the Domestic Environment. In: Proceedings of the ACM International Conference on Human-Robot Interaction - HRI 2006, p. 258 (2006)

8. Friedman, B., Kahn, P., Hagman, J.: Hardware companions?: What Online AIBO Discussion Forums Reveal About the Human-Robotic Relationship. In: Proceedings of the Conference on Human Factors in Computing Systems - CHI 2003, p. 273 (2003)

9. Galac, S., Knol, B.W.: Fear-Motivated Aggression in Dogs: Patient Characteristics, Diagnosis and Therapy. Animal Welfare 6(1), 9-15 (1997)

10. Hamill, L., Harper, R.: Talking Intelligence: a Historical and Conceptual Exploration of Speech-Based Human-Machine Interaction in Smart Homes. In: Proceedings of the International Symposium on Intelligent Environments, pp. 121-128 (2006)

11. Hyunsoo, S., Young-Min, K., Jong-Chan, P., Chong, H.K., Dong-Soo, K.: Design of a Robot Head for Emotional Expression: EEEX. In: International Symposium on Robot and Human Interactive Communication. RO-MAN, pp. 207-212 (2008)

12. Kang, S., Jaehong, K., Joochan, S.: A Robot Platform for Children and Its Action Control. In: International Conference on Control, Automation and Systems, pp. 2095-2098 (2008)

13. Kleiman, D.G.: Social Behavior of the Maned Wolf (Chrysocyon brachyurus) and Bush Dog (Speothos venaticus): A Study in Contrast. Journal of Mammalogy 53(4), 791-806 (1972)

14. Kriglstein, S., Wallner, G.: HOMIE. In: Proceedings of the International Conference Extended Abstracts on Human Factors in Computing Systems - CHI 2005, p. 2094 (2005) 
15. Lang, P., Bradley, B., Cuthbert, B.: International Affective Picture System (IAPS): Affective Ratings of Pictures and Instruction Manual. Technical Report A-8 (2008)

16. Lee, M.K., Makatchev, M.: How do People Talk with a Robot. In: Proceedings of the International Conference Extended Abstracts on Human Factors in Computing Systems CHI EA 2009. p. 3769 (2009)

17. Mehrabian, A.: Pleasure-Arousal-Dominance: A General Framework for Describing and Measuring Individual Differences in Temperament. Current Psychology 14(4), 261-292 (1996)

18. Michalowski, M.P., Šabanović, S., DiSalvo, C., Busquets, D., Hiatt, L.M., Melchior, N.A., Simmons, R.: Socially Distributed Perception: GRACE plays social tag at AAAI 2005. Autonomous Robots 22(4), 385-397 (2006)

19. Morris, J.D.: Observations: SAM: The Self-Assessment Manikin an Efficient CrossCultural Measurement of Emotional Response. Journal of Advertising Research 00218499, 63-68 (1995)

20. Norman, D.A.: Emotional Design: Why We Love (or Hate) Everyday Things. Basic Books (2004)

21. Picard, R.W.: Affective computing. The MIT Press, Cambridge (1997)

22. Pollick, F.E., Paterson, H.M., Bruderlin, A., Sanford, A.J.: Perceiving Affect from Arm Movement. Cognition 82(2), B51-B61 (2001)

23. Reeder, S., Kelly, L., Kechavarzi, B., Šabanović, S.: Breakbot. In: Proceedings of the ACM Conference on Designing Interactive Systems - DIS 2010, p. 61 (2010)

24. Russell, J.A.: A Circumplex Model of Affect. Journal of Personality and Social Psychology 39(6), 1161-1178 (1980)

25. Saerbeck, M., Bartneck, C.: Perception of Affect Elicited by Robot Motion. In: Proceedings of the ACM/IEEE International Conference on Human-Robot Interaction HRI 2010, pp. 53-60 (2010)

26. Schreiner, K.: Retail Robots on the Move. Intelligent Systems 16(2), 4-7 (2001)

27. Shibata, T., Yoshida, M., Yamato, J.: Artificial Emotional Creature for Human-Machine Interaction. In: IEEE International Conference on Systems, Man, and Cybernetics, Computational Cybernetics and Simulation, vol. 3, pp. 2269-2274 (1997)

28. Singh, A., Young, J.E.: Animal-Inspired Human-Robot Interaction: A Robotic Tail for Communicating State. In: Proceedings of the ACM/IEEE International Conference on Human-Robot Interaction - HRI 2012, pp. 237-238 (2012)

29. Staudte, M., Crocker, M.W.: The Utility of Gaze in Human-Robot Interaction. Metrics for Human-Robot Interaction (2008)

30. Wolftronix.com, http://www.wolftronix.com/tail/spine

31. Young, J.E., Sung, J., Voida, A., Sharlin, E., Igarashi, T., Christensen, H.I., Grinter, R.E.: Evaluating Human-Robot Interaction. International Journal of Social Robotics 3(1), 53-67 (2010)

32. Young, J.E., Xin, E., Sharlin, E.: Robot Expressionism Through Cartooning. In: Proceedings of the ACM/IEEE International Conference on Human-Robot Interaction HRI 2007, p. 309 (2007)

33. Young, J.E., Hawkins, E., Sharlin, E., Igarashi, T.: Toward Acceptable Domestic Robots: Applying Insights from Social Psychology. International Journal of Social Robotics 1(1), 95-108 (2008)

34. Young, J.E., Kamiyama, Y., Reichenbach, J., Igarashi, T., Sharlin, E.: How to Walk a Robot: A Dog-Leash Human-Robot Interface. In: International Symposium on Robot and Human Interactive Communication, RO-MAN, pp. 376-382 (2011)

35. Zecca, M., Endo, N., Momoki, S., Itoh, K., Takanishi, A.: Design of the Humanoid Robot KOBIAN - Preliminary Analysis of Facial and Whole Body Emotion Expression capabilities. In: International Conference on Humanoid Robots, pp. 487-492 (2008) 